\title{
Co-culture of bone marrow stromal cells and chondrocytes in vivo for the repair of the goat condylar cartilage defects
}

\author{
HAO SUN $^{1 *}$, YUE HUANG ${ }^{2 *}$, LEI ZHANG ${ }^{1}$, BIAO LI $^{1}$ and XUDONG WANG ${ }^{1}$ \\ ${ }^{1}$ Department of Oral and Cranio-Maxillofacial Surgery, Shanghai Ninth People's Hospital, School of \\ Medicine, Shanghai Jiao Tong University, Shanghai 200011; ${ }^{2}$ Department of Oral and Maxillofacial Surgery, \\ The First Affiliated Hospital of Fujian Medical University, Fuzhou, Fujian 350005, P.R. China
}

Received September 7, 2016; Accepted May 11, 2017

DOI: $10.3892 / \mathrm{etm} .2018 .6551$

\begin{abstract}
This study explored the feasibility of inducing the differentiation of BMSCs into chondrocytes through co-culture with chondrocytes in hydrogel constructs (Pluronic F-127 gel) in vivo for the repair of goat mandibular condylar cartilage defects. Chondrocytes and BMSCs were isolated from goat auricular cartilage and bone marrow, respectively, and were mixed at a ratio of 3:7. BMSCs were labelled with green fluorescence protein (GFP) using a retrovirus vector for tracing. Mixed cells were re-suspended in 30\% Pluronic F-127 at a concentration of $5 \times 10^{7}$ cells $/ \mathrm{ml}$ to form a gel-cell complex. The gel-cell complex was implanted into the temporomandibular joint condylar articular cartilage defects. The whole temporomandibular joint and adjacent tissues were harvested at 4, 8 , and 12 weeks after surgery, and gross observation, histology and collagen II expression were evaluated. In the co-culture group, cartilage-like tissues were formed, and abundant type II collagen could be detected by immunohistochemistry in the condylar cartilage defects. Confocal microscopy revealed that implanted GFP-labelled BMSCs were embedded in cartilage-like tissues. The co-culture system described herein provides a chondrogenic microenvironment to induce the chondrogenic differentiation of BMSCs in vivo without any additional cellular factors.
\end{abstract}

\section{Introduction}

Temporomandibular joint (TMJ) cartilage degeneration and defects caused by rheumatoid arthritis and osteoarthritis are

Correspondence to: Dr Xudong Wang, Department of Oral and Cranio-Maxillofacial Surgery, Shanghai Ninth People's Hospital, School of Medicine, Shanghai Jiao Tong University, 639 Zhi-zao-ju Road, Shanghai 200011, P.R. China

E-mail: xudongwang70@hotmail.com

*Contributed equally

Key words: bone marrow stromal cells, temporomandibular joint, condylar, articular common clinical problems, which can restrict opening of the mouth and cause TMJ locking and pain. Articular cartilage defects can be fully self-regenerated when the cartilage defect is $<3 \mathrm{~mm}$ in diameter (1); however, self-regeneration is limited in defects $>4 \mathrm{~mm}$ in diameter due to a lack of a proper chondrogenic niche (2). Current clinical treatments, including condylar surface shaving and periosteal grafts, are not adequately effective $(3,4)$. The repair of TMJ cartilage defects continues to perplex oral and maxillofacial surgeons.

Chondrocytes are one of the major cell types suitable for the repair of cartilage defects and may also have an essential role in the development and maintenance of the articular niche (5). Previous studies have demonstrated that mature articular chondrocytes can regenerate cartilage and steadily maintain the cartilage phenotype in subcutaneous environments $(6,7)$; however, the limited chondrocyte resources restrict the further utilization and generalization of these results.

Bone marrow stromal cells (BMSCs) have self-duplication capacity and possess the potential to differentiate into several tissues, including bone, cartilage, tendon, muscle, and fat $(8,9)$. BMSCs exist in bone marrow and can be harvested by a minimally invasive procedure. BMSCs can also be cultured in sufficient numbers in vitro while preserving their multipotential differentiation capacity (10). Therefore, BMSCs have become a popular focus of tissue engineering research.

It has been shown that BMSCs can maintain a high proliferative status in vitro (11). Therefore, previous investigations have focused on the cartilage formation induced from the BMSCs in vivo and in vitro (12). Yang et al (13) reported that the co-transplantation of BMSCs, chondrocytes, and chondrogenic factors into a hydrogel may enhance the chondrogenesis of BMSCs in subcutaneous environments, suggesting that chondrocytes may possess the potential to promote BMSC chondrogenesis. However, these studies adopted a large number of cellular factors to stimulate the differentiation of BMSCs into chondrocytes. The economic cost of the procedures used in these studies was great, and adverse effects may result. Liu et al (14) demonstrated that paracrine signalling by chondrocytes created a chondro-inductive niche similar to the articular subcutaneous environment used to direct the chondrogenesis of BMSCs in vitro. However, the co-culture of BMSCs and chondrocytes in the regeneration of condylar 
articular cartilage defects in vivo has not been reported until recently.

Pluronic F-127 (Poloxamer 407, PF-127) is a polyoxyethylene-polyoxypropylene surface active block copolymer. These gels exhibit reverse thermal behaviour and are therefore fluid at refrigerator temperature $\left(0-4^{\circ} \mathrm{C}\right)$ but are soft gels at body temperature (15). This characteristic has allowed PF-127 to be used as a carrier for various routes of administration, including oral (16), intranasal (17), rectal (18), and ocular (19).

In the present study, a combination of BMSCs and chondrocytes in hydrogel constructs (Pluronic F-127 gel) was used to repair goat TMJ articular cartilage defects. A green fluorescence protein (GFP) retrovirus vector was used to track the presence of BMSCs in vivo and to evaluate the possibility of using BMSCs and chondrocytes for cartilage defect repairs in vivo to develop a novel tissue engineering approach for the clinical reconstruction of TMJ cartilage.

\section{Materials and methods}

Animal model. All procedures followed the ethical guidelines of the Animal Experimental Ethical Inspection of Shanghai Ninth People's Hospital affiliated to Shanghai Jiao Tong University (Shanghai, China). A total of 12 mature male healthy goats, aged 6-8 months and weighing 10-22 kg were purchased from Shanghai Agricultural Institute (Shanghai, China) and subsequently randomly divided into a gel-cell group (group 1) and a gel alone group (control; group 2; Table I).

Cell source and culture conditions. Mature goats were given intramuscular injections of ketamine-846 (Institute of Animal Science, Changchun University of Agriculture and Animal Science, Changchun, China.) anaesthesia mixture prior to bone marrow harvesting. Bone marrow cells were harvested from the ilium by a sterile surgical technique and suspended in $1 \mathrm{X}$ phosphate-buffered saline (PBS) containing $2 \%$ bovine serum albumin (BSA). Following centrifugation (179 $\mathrm{x} g$ at room temperature) for $5 \mathrm{~min}$ to remove serum ingredients, bone marrow cells were treated with sterile water for $10 \mathrm{sec}$ to disrupt red blood cells, re-suspended in 2\% BSA in PBS, and centrifuged (179 x g at room temperature) again for 5 min. Precipitated bone marrow cells from each goat were cultured in 100-mm diameter tissue culture dishes with $10 \mathrm{ml} \alpha$-minimum essential medium (Gibco; Thermo Fisher Scientific, Inc., Waltham, MA, USA) supplemented with $10 \%$ foetal bovine serum (FBS) (Gibco; Thermo Fisher Scientific, Inc.) and ampicillin antibiotic at $37^{\circ} \mathrm{C}$ in a humidified atmosphere containing $5 \% \mathrm{CO}_{2}$ for 1 week. Once the attached cells formed a large colony, the cells were transferred to a new plate for expansion until $80 \%$ confluence was achieved.

BMSCs were labelled with green fluorescence protein (GFP), as previously described (13). GFP lentiviruses were produced by transient transfection into the 293FT cell line (Invitrogen; Thermo Fisher Scientific, Inc.) with the pLenti6.2-GW/EmGFP vector and three packaging plasmids, pLP1, pLP2, and pLP/VSVG (Invitrogen; Thermo Fisher Scientific, Inc.).

For the isolation of auricular chondrocytes, auricular cartilage $\left(3 \times 3 \mathrm{~cm}^{2}\right)$ was harvested from the goat ear by a sterile surgical technique and cut into $2 \times 2 \mathrm{~mm}^{2}$ sections. The tissue was then digested by $0.25 \%$ pancreatic enzyme for $30 \mathrm{~min}$, followed by digestion with $0.1 \%$ collagenase II for $4 \mathrm{~h}$ at $37^{\circ} \mathrm{C}$. Following filtration, cells were centrifuged $(179 \mathrm{x} g$ at room temperature) for $5 \mathrm{~min}$ and re-suspended in $1 \mathrm{X}$ PBS containing $2 \%$ BSA. The auricular chondrocytes from each goat were cultured in 100-mm diameter tissue culture dishes with $10 \mathrm{ml}$ 10\% FBS Dulbecco's modified Eagle medium.

Surgical procedures. Goats were administered general intramuscular anaesthesia using ketamine-846 anaesthesia mixture prior to harvesting condylar cartilage. An incision was made near the ear side to expose the article area and the cartilage on the surface of the condylar process (Fig. 1). The full-thickness condylar cartilage was totally removed to create a lesion in the condyle surface using a surgical drill (Fig. 1).

Chondrocytes and GFP-labelled BMSCs were also collected from the culture and mixed using a ratio of 3:7 (chondrocytes:BMSCs), as previously described by our group (14). Mixed cells were re-suspended in 30\% Pluronic F-127 at a density of $5 \times 10^{7}$ cells $/ \mathrm{ml}$ to form a gel-cell complex. Pluronic F-127 gel mixed with chondrocytes and BMSCs was implanted into both sides of the condylar cartilage defects (Fig. 1). Pluronic F-127 gel alone was implanted into the condylar cartilage defects of group 2 as a control. Articular capsules were adequately closed. All goats were allowed to move freely after these procedures and housed in separated cages, fed soft food pellets ad libitum, and kept in a temperature-controlled environment with a 12-h light/dark cycle.

Gross morphology and X-ray observations. At 4, 8, and 12 weeks after surgery, the maximal passive mouth-opening range under general intramuscular anaesthesia was measured. Four goats in both groups were X-rayed and subsequently sacrificed for gross morphology observation. The TMJ was exposed, and the whole joint and adjacent tissues were harvested for gross morphology and histology analyses.

Histology. After evaluating the gross morphology, the TMJ samples were fixed in $10 \%$ neutral formalin buffer for 1 week at room temperature and decalcified in $30 \%$ formic acid for $\sim 2$ weeks. The samples were then dehydrated and embedded into paraffin blocks.

Samples were cut into $5-\mu \mathrm{m}$ sections and stained with hematoxylin and eosin, as previously described (8). Sections were viewed under a light microscope and graded semi-quantitatively, using a scoring system modified from that described by Wakitani et al (20). The scale is composed of five categories and assigns a score ranging from 0 to 14 points (Table II). The distribution of GFP-labelled cells was observed and captured by laser scanning spectral confocal microscopy (TCS SP5; Leica Microsystems Wetzlar, Maneheim, Germany). The inflammatory response (inflammatory cells, fibroblasts) and osteophyte formation were also evaluated using histological slides.

Detection of GFP-labelled cells in mixed cell tissues. Chondrocytes and BMSCs (labelled with GFP) were seeded together in $30 \%$ Pluronic F-127 at a ratio of 3:7 at a density of $5 \times 10^{7}$ cells $/ \mathrm{ml}$ to form a gel-cell complex. The tissues containing GFP-labelled BMSCs were collected, frozen in 
Table I. Animal grouping.

\begin{tabular}{lcccc}
\hline Group & 4 weeks & 8 weeks & 12 weeks & Total \\
\hline Gel-cell complex & 2 & 2 & 2 & 6 \\
Gel only & 2 & 2 & 2 & 6 \\
\hline
\end{tabular}
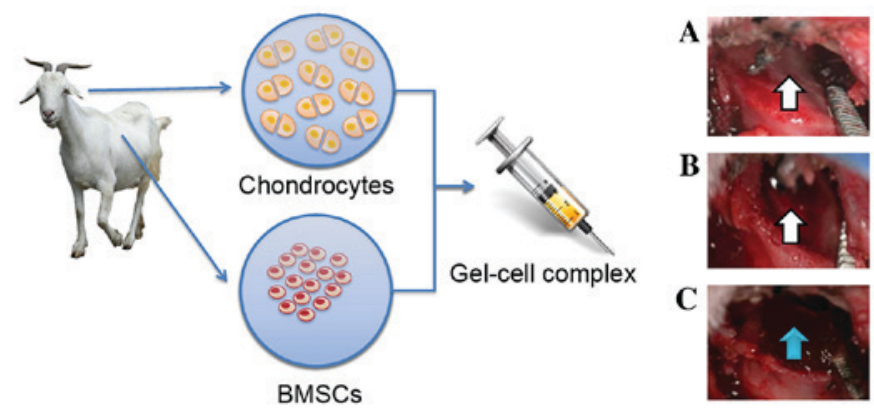

Figure 1. Experimental schematic. Chondrocytes and BMSCs were isolated from goat auricular cartilage and bone marrow, respectively, mixed with $30 \%$ Pluronic F-127 to form a gel-cell complex and implanted into the TMJ condylar articular cartilage defects. (A and B) The white arrow indicates that the full-thickness condylar cartilage was totally removed and that a large hole was created in the condyle surface. (C) The blue arrow indicates that the gel-cell complex was implanted into the condylar cartilage defects. BMSCs, bone marrow stromal cells; TMJ, temporomandibular joint.

optimum cutting temperature gel, and sliced at a thickness of $5 \mu \mathrm{m}$. The exact distribution of the labelled cells was observed and captured by a Leica TCS SP5 laser scanning spectral confocal microscope. The chondrogenic differentiation of the labelled cells was further confirmed by histological analysis.

Immunohistochemical staining. Following de-paraffinisation and an endogenous peroxidase block, the sections were heated in a water bath at $98^{\circ} \mathrm{C}$ with $0.01 \mathrm{M}$ citrate buffer $(\mathrm{pH}, 6.0)$ for $20 \mathrm{~min}$, treated with $1 \% \mathrm{H}_{2} \mathrm{O}_{2}$ in water for $30 \mathrm{~min}$, incubated with mouse monoclonal antibody to type II collagen (Neomarkers, Fremont, CA, USA) at a dilution of 1:5,000 overnight at $4^{\circ} \mathrm{C}$, and visualized using a 3,3'-diaminobenzidine (DAB) detection kit (Dako; Agilent Technologies, Inc., Santa Clara, CA, USA). Negative controls were prepared using PBS instead of antibody.

Statistical analysis. Statistical analysis was performed using Student's t-test to assess the differences between the two groups. Estimates were given as medians, and two-tailed values of $\mathrm{P}<0.05$ were considered to indicate a statistically significant difference.

\section{Results}

Preparation of BMSCs and chondrocytes. Newly isolated BMSCs began to adhere within $24 \mathrm{~h}$ after incubation on a $10-\mathrm{cm}$ culture dish. Several cell colonies were formed after 5 days of culture. BMSCs exhibited spindle-like or elongated shapes (Fig. 2A). Newly isolated chondrocytes were polygonal in shape with more regular dimensions. Cells were shown to attach to the dish within 3 days (Fig. 2B).
Gross assessment and X-ray observation. As outlined in Fig. 3, no infection was observed in any of the TMJ joints from either group. In the gel-cell transplanted group, the passive mouth-opening range of the experimental animals 4 weeks after surgery was similar to their pre-operative states. No adhesions were found between the cartilage defects of the condylar processes and the articular discs. However, the condylar process surfaces were slightly rough and depressed (Fig. 3A). At 8 weeks post-surgery, the passive mouth-opening range remained similar to the pre-operative levels. No further adhesions were found in the gap between cartilage defect areas and the articular discs. The condylar process surfaces became smooth, and a cartilage-like layer was observed on the condylar process surfaces (Fig. 3B). At 12 weeks post-surgery, the mouth-opening range showed no notable change compared to pre-operative levels. The condylar process surface of each goat was smooth, and more cartilage-like layers were observed on the condylar process surfaces (Fig. 3C). The X-ray film analysis showed that there was no obvious bone destruction and no osteophyte was formed (Fig. 3D-F).

In the gel alone group, the passive mouth-opening range of the goat was slightly shorter than the pre-operative levels at 4 weeks post-surgery. Adhesive tissue was observed between the cartilage defect areas of the condylar process and the articular disc. The condylar process surface was rough and depressed. No Pluronic F-127 gel was found on the defect areas (Fig. 3G). At 8 weeks post-surgery, mouth opening was much shorter compared to both the pre-operative levels and the levels 4 weeks post-surgery. More adhesive tissue was found between the cartilage defect areas and articular discs compared to 4 weeks post-surgery. The rough condylar process surface remained the same (Fig. $3 \mathrm{H}$ ). At 12 weeks post-surgery, the animal mouth-opening range was even shorter than that observed at 8 weeks post-surgery. Severe adhesions were observed between the cartilage defect areas and articular discs. However, some fibrous-like tissues were observed on the rough condylar process surfaces (Fig. 3I). X-ray film analysis showed that the joint space seem broadened compared with the gel-cell group (Fig. 3J-L). These results suggested that the cartilage defect healing process was delayed in the goats exposed to gel alone.

Histological evaluation. As outlined in Fig. 4, in the gel-cell group after hematoxylin and eosin staining, the condylar process surfaces were slightly rough and filled with cartilaginous and fibrous tissue at 4 weeks post-surgery (Fig. 4A). At 8 weeks, 70\% of the defect areas were filled with cartilaginous and fibrous tissue (Fig. 4B). At 12 weeks, the surfaces became smooth and the superficial layers were repaired with cartilage-like tissue, while the deeper layers were replaced with bone or osseous-like tissue (Fig. 4C).

In the gel only control group, the surface tissue of the defect areas in the condylar processes were disorganised and filled with a small amount of fibrous tissue at 4 weeks post-surgery (Fig. 4D). However, no Pluronic F-127 gel was left on the defect areas, indicating that the gel had degraded by that time. At 8 weeks, the surfaces of the defects were still rough; $\sim 60 \%$ of the defects were filled with fibrous or osseous-like tissue (Fig. 4E). At 12 weeks, the surfaces were filled with fibro-like tissue without any cartilaginous tissue (Fig. 4F). 
Table II. Histological grading scale for the cartilage defects.

\begin{tabular}{lc}
\hline Category & Points \\
\hline Cell morphology & \\
Hyaline cartilage & 0 \\
Mostly hyaline cartilage & 1 \\
Mostly fibrocartilage & 2 \\
Mostly non-cartilage & 3 \\
Non-cartilage only & 4 \\
Matrix-staining (metachromasia) & \\
Normal (compared with host adjacent cartilage) & 0 \\
Slightly reduced & 1 \\
Markedly reduced & 2 \\
No metachromatic stain & 3 \\
Surface regularity & \\
Smooth $(>3 / 4)$ & 0 \\
Moderate $(>1 / 2-3 / 4)$ & 1 \\
Irregular $(1 / 4-1 / 2)$ & 2 \\
Severely irregular $(<1 / 4)$ & 3 \\
Thickness of cartilage & \\
$>2 / 3$ & \\
$1 / 3-2 / 3$ & 0 \\
$<1 / 3$ & 1 \\
Integration of donor with host adjacent cartilage & \\
Both edges integrated & \\
One edge integrated & \\
Neither edge integrated & \\
Total maximum & 0 \\
\hline
\end{tabular}

Histological grading of the repair tissue. Scores of group 1 were improved at 8 and 12 weeks compared with those at 4 weeks (Table III). These results were in accordance with those of macroscopic and histological observations. The scores of the experimental groups were similar, with the exception of surface regularity. In group 2, the histological scores indicated markedly inferior repair, when compared with group 1.

Immunohistochemical staining. To determine if the newly formed tissue was cartilage, immunohistochemical staining was performed using sections harvested at 4, 8 and 12 weeks post-surgery. In the gel-cell complex group, newly formed surface layers exhibited weak staining of type II collagen at 4 weeks, indicating that the cells in this layer had become chondrocytes and started to secrete type II collagen (Fig. 4G). At 8 and 12 weeks, the cartilage layer demonstrated strong staining of type II collagen (Fig. 4H and I, respectively), suggesting that more cartilage had formed. No type II collagen staining was observed in the gel alone group at any of the three time points, indicating that no cartilage formed in the gel alone group (Fig. 4J-L).

GFP-labelled cells in mixed cell engineered tissues. BMSCs were successfully infected and labelled with GFP (Fig. 5A). GFP-labelled cells were detected in the newly formed cartilage lacuna of the newly formed tissue at 12 weeks post-surgery in gel-cell complex-transplanted goats, using laser confocal microscopy (Fig. 5B), which provided direct evidence that the BMSCs had been transformed into chondrocyte-like cells. No GFP-positive cells were observed in the control group. These results further indicate that the chondrogenic niche accelerates the transition of BMSCs to chondrocytes in the TMJ environment.

\section{Discussion}

Despite its remarkable ability to resist mechanical loading, articular cartilage is not capable of mounting a useful reparative reaction in response to damage (21). Among the most common causes of damage to cartilage are trauma, osteoarthritis and osteochondritis dissecans (22). Each of these three conditions can present in similar ways, with pain, swelling and impaired movement of the joint. Avascularity is a major factor in the poor cartilage repair response; this means that there is no supply of clotting materials or cells to produce repair material following damage or insult (23). Avascularity may also indicate that there is no supply of mesenchymal stem cells.

For a long time, it has been the goal of surgeons to develop a reliable method to repair damaged articular cartilage. Techniques have ranged from debris removal techniques, such as debridement and lavage developed in the 1940s, to osteochondral transplant techniques, marrow stimulation techniques and the latest generation of cell-based tissue engineering techniques, such as autologous chondrocyte implantation (24). Bone marrow stimulation techniques are based on the principle that when natural full-depth defects cross the subchondral bone, bleeding from the bone marrow leads to the production of repair tissue within the lesion (25). The problem with these techniques is that, although the clot produced fills the defect and produces repair tissue, this tissue is mostly fibro-cartilaginous and is a poor mechanical substitute for the natural hyaline cartilage (26).

Difficulties associated with producing hyaline cartilage-like repair tissue in defects using these surgical techniques, combined with the problems associated with revision surgery of total joint replacements in young active individuals, has led to the expansion of tissue engineering approaches to treat damaged cartilage. Currently, the only cell-based tissue engineering approach that is licenced for use in patients is autologous chondrocyte implantation (ACI). This procedure, which was pioneered by Brittberg and first used in 1987, involves the use of autologous chondrocytes to produce repair tissue within a defect (27). ACI has been a successful technique, and even the first generation used in the late $1980 \mathrm{~s}$ produced improvements in joint function, reduced pain scores, and in some cases, produced hyaline-like repair tissue (28). Despite this success, ACI has had and continues to have problems. The first is the risk of donor site morbidity, this has been partially dealt with by moving away from the use of periosteal flaps but still remains a problem at the cartilage harvest site. Damage to the cartilage can lead to further degeneration and osteoarthritis over the long term (29). Another major drawback with $\mathrm{ACI}$ is the in vitro cell culture stage. When chondrocytes are cultured in a $2 \mathrm{D}$ environment for an extended period of time, they dedifferentiate. Dedifferentiation involves a decrease in 
A

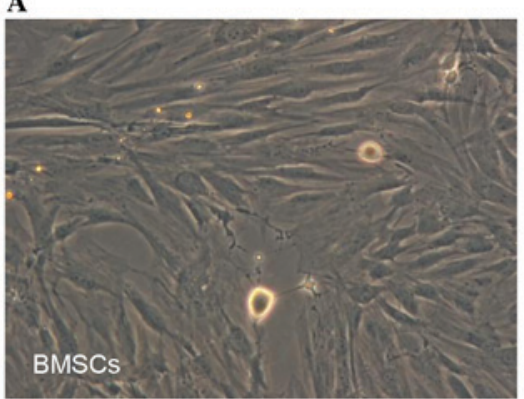

B

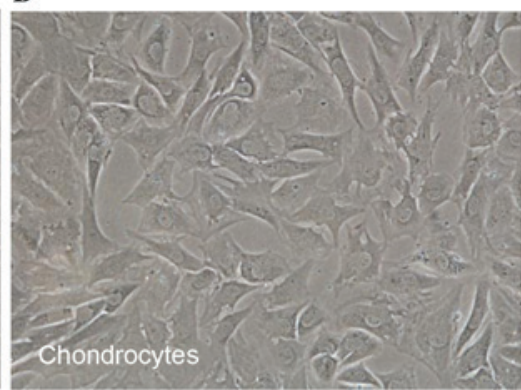

Figure 2. Characteristics of BMSCs and chondrocytes. BMSCs began to adhere within $24 \mathrm{~h}$ after inoculation. (A) BMSCs were spindle-shaped or scalene triangle-shaped (phase contrast, x100). Newly isolated chondrocytes were rounded, with strong reflection. Cells were adherent to the dish within 3 days. (B) Adhered cells were shaped like transparent polygons with great refractivity (phase contrast, $\mathrm{x} 100$ ).

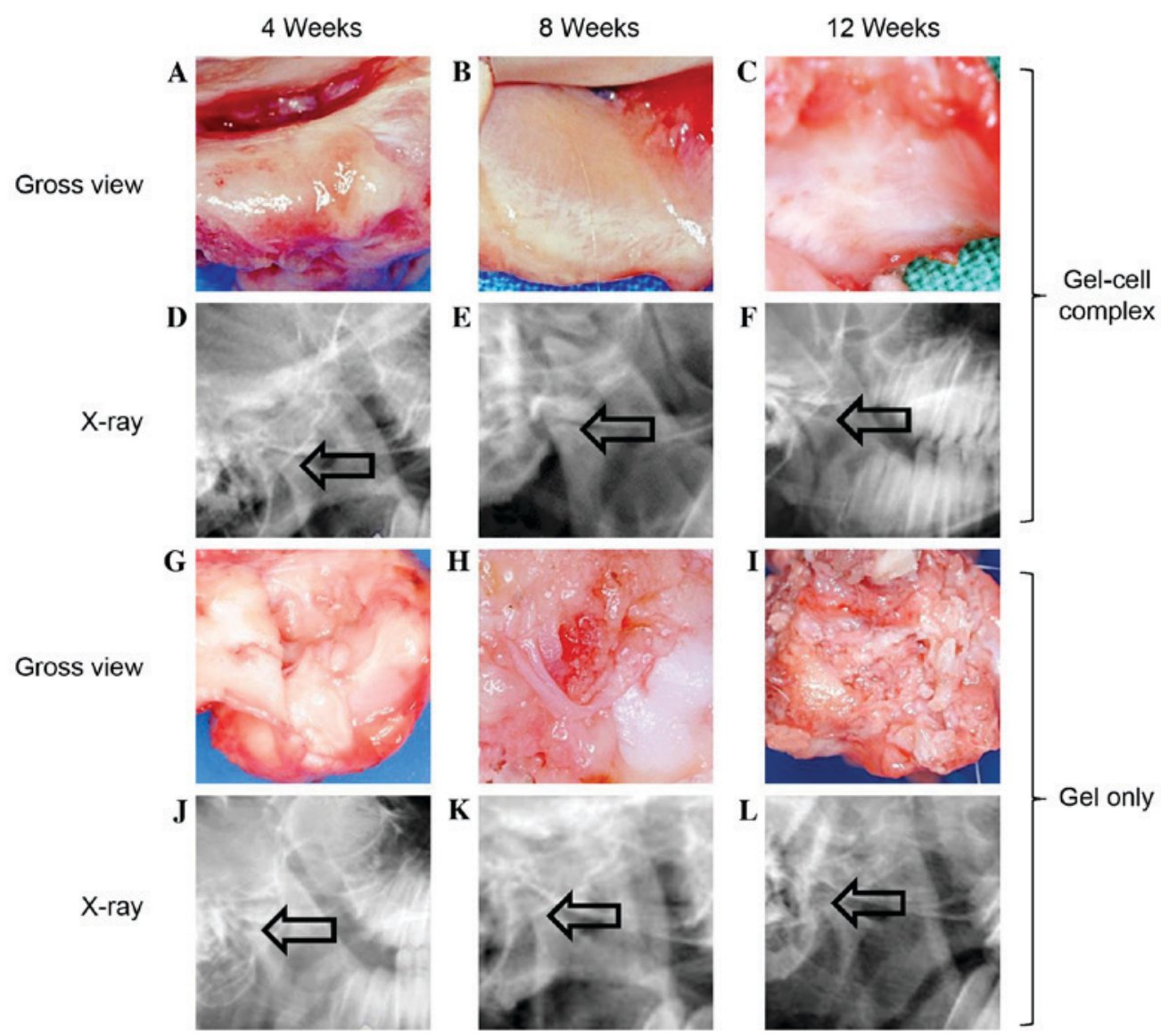

Figure 3. Monitor repaired defects post-surgery by gross view and x-ray. In gel-cell complex implantation group: (A) At week 4, the surfaces were slightly rough and depressed in gross view, and no Pluronic F-127 gel was found on the defect areas (a); (B) at week 8, the surfaces were smooth and a cartilage-like layer can be observed on the surface; (C) at week 12, the surfaces were smooth and a cartilage-like layer can be observed on the surface. (D-F) The X-ray films showed that there was no obvious bone destruction and no osteophyte was formed. In the gel only group: (G) The surfaces were slightly rough and depressed, and no Pluronic F-127 gel was found; (H) the surfaces were rough and depressed; (I) the surfaces were rough and had some fibrous-like tissues on the surface. (J-L) The X-ray films showed that there was no obvious bone destruction and no osteophyte was formed. In the gel only group, the joint space seemed broadened compared with the gel-cell group (indicated by the arrow, phase contrast, x100).

the expression of collagen markers, such as type II collagen, and an increase in type I collagen production; the cells also develop a fibroblastic morphology (30). This process prevents chondrocytes cultured for too long in vitro from being able to produce repair cartilage. The very low cell density in cartilage and the small areas available for harvesting, combined with their limited ability for useful expansion in vitro, means that ACI can only utilise a particularly small number of cells, whereas a larger number may have more success in producing hyaline-like cartilage repair tissue as cell-to-cell contact is believed to be important for chondrogenesis (31). There is a wide range of different approaches that are used to surgically treat damaged or diseased cartilage, ranging from marrow stimulation and debridement to cell-based tissue engineering in the form of ACI. However, each of the aforementioned techniques has faults, as well as benefits, so other avenues 


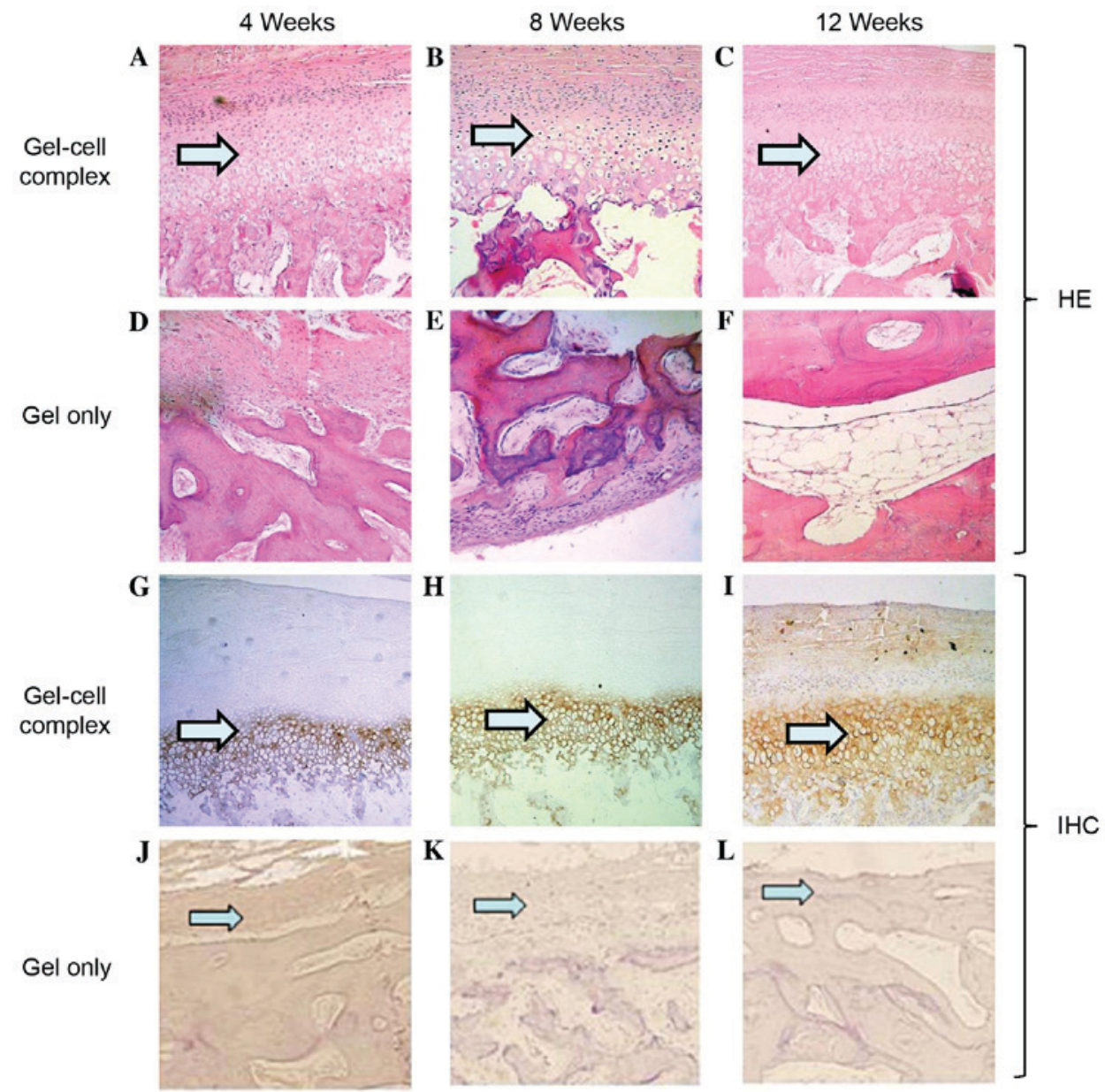

Figure 4. Histological evaluation of defects via HE staining and IHC staining with collagen II. For HE staining in the gel-cell complex group: (A) At week 4, the surfaces were slightly irregular and filled with cartilaginous and fibrous tissue; (B) at week 8 , the surfaces were much smoother, and most parts of the defects were filled with cartilaginous and fibrous tissue; (C) and at week 12, the surface became very smooth, and the superficial layer was repaired with cartilage and the deeper layer was remodelled with bone or osseous-like tissue. For HE staining in the gel only group: (D) At week 4, the surfaces were irregular and filled with fibrous tissue but not cartilaginous and/or osseous tissue; (E) at week 8, the surfaces were still irregular, and most parts of the defects were filled with fibrous or osseous-like tissue; (F) at week 12, the surfaces were smooth and repaired with mostly osseous-like tissue and some fibrous tissue without any cartilage tissue (phase contrast, x100). For IHC staining in the gel-cell complex group: (G) At week 4, the cartilage layer was stained with type II collagen at a low intensity; (H) at week 8, the cartilage layer was stained with type II collagen at a much higher intensity; (I) at week 12, the cartilage layer was stained with type II collagen at a high intensity. For IHC staining in the gel only group: (J) At week 4, type II collagen staining was negative, and no Pluronic F-127 gel was found on the surface. The same was observed at (K) 8 weeks and (L) 12 weeks post-surgery. HE, hematoxylin and eosin; IHC, immunohistochemical.

are being explored to treat damaged cartilage. One of these avenues is the use of mesenchymal stem cells (MSCs), rather than chondrocytes, in tissue engineering applications (32).

The broad definition of an MSC is often given as a culture-adherent multipotent progenitor cell that can differentiate down the adipogenic, chondrogenic and osteogenic lineages (33). As well as being found in various different tissue types, under the correct conditions, MSCs are capable of producing a wide range of tissues in vivo and in vitro. By definition, MSCs can be differentiated into cartilage, bone and fat-producing cells in vivo and in vitro and it is this feature that makes them such a potentially powerful tool within tissue engineering. To induce chondrogenesis in MSCs, the cells need to be in close contact, as well as exposed to the correct soluble factors (31). To achieve this, MSCs are suspended in culture medium and spun in a centrifuge to produce a pellet culture. The pellet is then cultured in a growth medium containing tumor growth factor (TGF)- $\beta$, leading to the development of cartilaginous tissue that stains for toluidine blue and contains type II collagen (34). A problem with this cartilage model, however, is that TGF- $\beta$-induced chondrogenesis over time leads to the hypertrophy of chondrogenic MSCs and an increased expression of osteogenic markers, such as type $\mathrm{X}$ collagen and Runx2, in a similar progression of differentiation to that observed during bone formation via endochondral ossification, in which chondrocytes undergo hypertrophy leading to apoptosis calcification (35). MSCs maintain their chondrogenic ability, even throughout long-term monolayer culture, although their replicative capacity is not infinite. Banfi et al (36) estimated that the useful clinical limit for expansion would be 17 population doublings, more than twice as many as chondrocytes can be usefully expanded by.

In the present study, BMSCs and chondrocytes were co-cultured in vitro at ratios of 6:4 or 7:3 and the findings showed that chondrocytes provided a chondrogenic micro-environment to induce chondrogenic differentiation of BMSCs and thus promote the in vitro chondrogenesis of BMSCs. It was also demonstrated that, at a ratio of 1:4, chondrocytes with BMSCs 
Table III. Results of the histological grading scale.

\begin{tabular}{lcccccrr}
\hline & \multicolumn{7}{c}{ Grade } \\
\cline { 3 - 7 } Group & $\begin{array}{c}\text { Time } \\
\text { (weeks) }\end{array}$ & $\begin{array}{c}\text { Cell } \\
\text { morphology }\end{array}$ & $\begin{array}{c}\text { Matrix } \\
\text { staining }\end{array}$ & $\begin{array}{c}\text { Surface } \\
\text { regularity }\end{array}$ & $\begin{array}{c}\text { Thickness } \\
\text { of cartilage }\end{array}$ & Integration & Total \\
\hline Gel-cell complex & 4 & 2.0 & 1.0 & 0.7 & 1 & 0.0 & 4.7 \\
& 8 & 2.0 & 1.0 & 0.0 & 1 & 0.0 & 4.0 \\
Gel only & 12 & 2.0 & 1.0 & 0.0 & 1 & 0.0 & 4.0 \\
& 4 & 3.5 & 2.5 & 1.5 & 2 & 1.5 & 11.0 \\
& 8 & 3.0 & 3.0 & 2.0 & 2 & 2.0 & 12.0 \\
& 12 & 4.0 & 3.0 & 2.5 & 2 & 2.0 & 13.5 \\
\hline
\end{tabular}

A

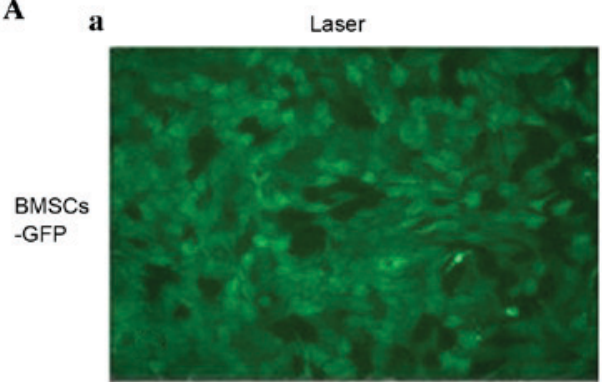

b

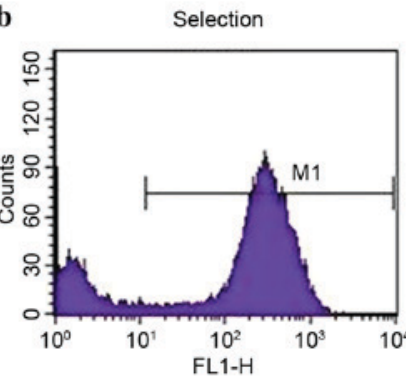

Selection
File:selection

Acquisition date:06-nov-06

Marker \% Gated

M1 78.81
B

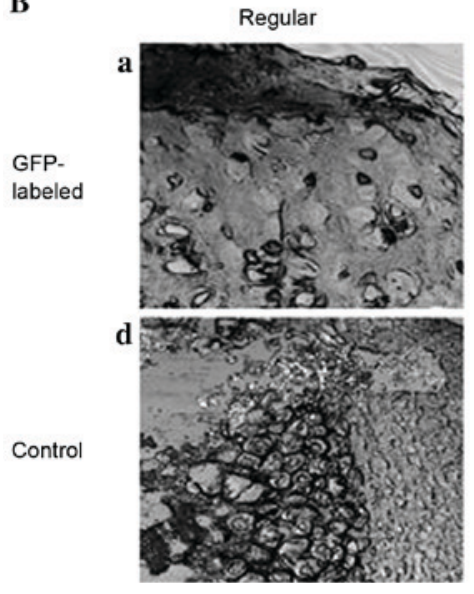

Laser

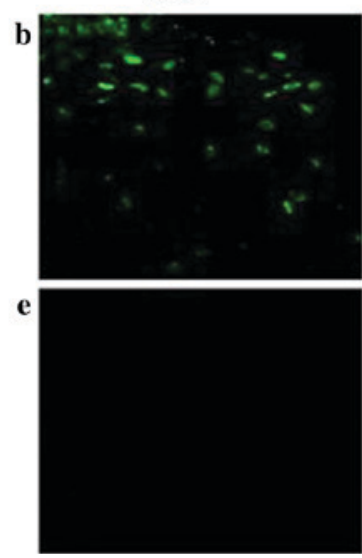

Merge
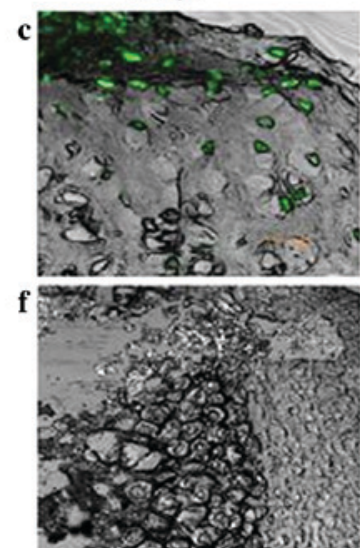

Figure 5. Evaluation of cartilage repairby confocal microscopy. (A) Quanfication of GFP expression in BMSCs. (a) GFP expression distribution of BMSCs in fluorescent microscopy (x100). (b) GFP-expressed BMSCs selected by flow cytometry. (B) Repaired cartilage comparison between gel-cell complex implantation and control groups were demonstrated by (a and b) confocal microscopy (x200, (c and d) bright field images and (e and f) merged images. GFP-expressed BMSCs were evenly distributed in repaired cartilage area and formed typical lacuna structures at 12 weeks post-surgery. GFP, green fluorescent protein; BMSCs, bone marrow stromal cells.

provided a chondrogenic microenvironment (37). In this experiment to repair condylar defects, BMSCs and chondrocytes were mixed using a ratio of 7:3 in Pluronic F-127 without any growth factors. The condylar surface became very smooth, the superficial layer was repaired with cartilaginous tissue, and the deeper layer was remodelled with bone or osseous-like tissue at 12 weeks after surgery. Cartilaginous tissue was formed at 4, 8 and 12 weeks after surgery, as shown by the staining of type II collagen. The mouth-opening range of the experimental group was similar to pre-operative levels, and no adhesions were found between the cartilage defect areas and the articular discs at 4 , 8 and 12 weeks after surgery. These data suggest that mixed
BMSCs and chondrocytes enhance the healing of articular cartilage defects.

There are several possible explanations for the manner whereby BMSCs and chondrocytes interact with each other in osteochondral repair. Several types of soluble factors secreted by chondrocytes, such as TGF- $\beta$ s, IGF-1 and BMPs, have a direct effect on BMSCs by inducing the differentiation of BMSCs into chondrocytes (37). In addition, chondrocytes synthesize and secrete cartilage-specific extracellular matrix to induce BMSC differentiation into chondrocytes (38). BMSCs differentiate into chondrocytes by cell-to-cell contact signalling between BMSCs and chondrocytes. Finally, chondrocytes can secrete 
anti-angiogenic factors to prevent vascular invasion and retain their own phenotypes, thus preventing neo-vascularization and ossification (39). The findings of this study also showed no vascular invasion at the superficial and the deeper layers of the condylar surface at 12 weeks after surgery.

In the present study, goats were implanted with BMSCs labelled with GFP, and thus, it was possible to trace the implanted BMSCs and to further illustrate the differentiation and distribution of BMSCs post-surgery. As shown in our results, the GFP-labelled cells were detected in newly formed cartilage lacunae of repaired tissue at 12 weeks post-surgery, which provides convincing evidence that the implanted BMSCs were able to differentiate into mature chondrocytes in the chondrocyte-mediated chondro-inductive niche in the TMJ environment. These results also indicate that the implanted BMSCs were a vital cell source of newly formed articular tissue.

Another precondition for a cartilage regeneration system is suitable matrices that can be used as scaffold frameworks for cell viability and proliferation while maintaining the original cellular phenotype. Investigating biomaterials used as three-dimensional scaffolds for cell delivery and therapy has recently become a major focus in the field of tissue engineering $(40,41)$.

The culture of autologous chondrocytes has previously been used to induce proliferation and differentiation in a hydrogel system (42). Fully thermo-reversible gelling polymers have attracted considerable attention for use as scaffolds to hold cells in situ during cartilage formation $(43,44)$. These thermo-reversible polymers can revert from a solid to a liquid state and from a liquid to a solid state without losing their intrinsic properties. Additionally, these polymers are fully soluble in aqueous solutions at temperatures below their lower critical solution temperature (LCST) but solidify to form a hydrated gel at temperatures above their LCST (45). Although many studies have been conducted to evaluate thermo-reversible hydrogels for use as injectable scaffolds, few in vivo cartilage tissue engineering tests involving differentiated materials have been conducted.

In this study, Pluronic F-127 gel provided suitable three-dimensional scaffolds for the BMSC and chondrocyte co-culture system. GFP-labelled BMSCs were detected in the newly formed cartilage lacuna of the newly formed tissue in gel-cell transplanted goats, which provided direct evidence that the BMSCs had been transformed into chondrocyte-like cells.

In conclusion, the present findings have demonstrated the possibility of using BMSCs and chondrocytes for the osteochondral repair of TMJ in vivo, which offer a novel treatment for the clinical reconstruction of TMJ. However, the exact mechanism whereby chondrocytes promote chondrogenic differentiation of BMSCs remains to be addressed.

\section{Acknowledgements}

This project was financially supported in part by the National Science Foundation of China (grant no. 81271122), the Interdisciplinary Program of Shanghai Jiao Tong University (grant nos. YG2017ZD03 and YG2014QN02), the Research Fund of Science and Technology Commission of Shanghai City (grant no. 18410712000), and the Shanghai Municipal Education Commission-Gaofeng Clinical Medicine Grant Support (grant no. 20152225).

\section{References}

1. Kim HK, Moran ME and Salter RB: The potential for regeneration of articular cartilage in defects created by chondral shaving and subchondral abrasion. An experimental investigation in rabbits. J Bone Joint Surg Am 73: 1301-1315, 1991.

2. Convery FR, Akeson WH and Keown GH: The repair of large osteochondral defects. An experimental study in horses. Clin Orthop Relat Res 82: 253-262, 1972.

3. Valentini V, Vetrano S, Agrillo A, Torroni A, Fabiani F and Iannetti G: Surgical treatment of TMJ ankylosis: Our experience (60 cases). J Craniofac Surg 13: 59-67, 2002.

4. Hikiji H, Takato T, Matsumoto S and Mori Y: Experimental study of reconstruction of the temporomandibular joint using a bone transport technique. J Oral Maxillofac Surg 58: 1270-1277, 2000.

5. Görtz S and Bugbee WD: Allografts in articular cartilage repair. J Bone Joint Surg Am 88: 1374-1384, 2006.

6. Hangody L, Feczkò P, Bartha L, Bodò G and Kish G: Mosaicplasty for the treatment of articular defects of the knee and ankle. Clin Orthop Relat Res (391 Suppl): S328-S36, 2001.

7. Jamali AA, Emmerson BC, Chung C, Convery FR and Bugbee WD: Fresh osteochondral allografts: Results in the patellofemoral joint. Clin Orthop Relat Res: 176-185, 2005.

8. Liechty KW, MacKenzie TC, Shaaban AF, Radu A, Moseley AM, Deans R, Marshak DR and Flake AW: Human mesenchymal stem cells engraft and demonstrate site-specific differentiation after in utero transplantation in sheep. Nat Med 6: 1282-1286, 2000.

9. Minguell JJ, Erices A and Conget P: Mesenchymal stem cells. Exp Biol Med (Maywood) 226: 507-520, 2001.

10. Pittenger MF, Mackay AM, Beck SC, Jaiswal RK, Douglas R, Mosca JD, Moorman MA, Simonetti DW, Craig S and Marshak DR: Multilineage potential of adult human mesenchymal stem cells. Science 284: 143-147, 1999.

11. Baksh D, Yao R and Tuan RS: Comparison of proliferative and multilineage differentiation potential of human mesenchymal stem cells derived from umbilical cord and bone marrow. Stem Cells 25: 1384-1392, 2007.

12. Li WJ, Tuli R, Huang $X$, Laquerriere $P$ and Tuan RS: Multilineage differentiation of human mesenchymal stem cells in a three-dimensional nanofibrous scaffold. Biomaterials 26: 5158-5166, 2005.

13. Yang HN, Park JS, Na K, Woo DG, Kwon YD and Park KH: The use of green fluorescence gene (GFP)-modified rabbit mesenchymal stem cells (rMSCs) co-cultured with chondrocytes in hydrogel constructs to reveal the chondrogenesis of MSCs. Biomaterials 30: 6374-6385, 2009.

14. Liu X, Sun H, Yan D, Zhang L, Lv X, Liu T, Zhang W, Liu W, Cao Y and Zhou G: In vivo ectopic chondrogenesis of BMSCs directed by mature chondrocytes. Biomaterials 31: 9406-9414, 2010.

15. Bohorquez M, Koch C, Trygstad T and Pandit N: A study of the temperature-dependent micellization of pluronic F127. J Colloid Interface Sci 216: 34-40, 1999.

16. Padilla M, Clark GT and Merrill RL: Topical medications for orofacial neuropathic pain: A review. J Am Dent Assoc 131: 184-195, 2000.

17. Ved PM and Kim K: Poly(ethylene oxide/propylene oxide) copolymer thermo-reversible gelling system for the enhancement of intranasal zidovudine delivery to the brain. Int J Pharm 411: $1-9,2011$.

18. Barichello JM, Morishita M, Takayama K, Chiba Y, Tokiwa S and Nagai T: Enhanced rectal absorption of insulin-loaded Pluronic F-127 gels containing unsaturated fatty acids. Int J Pharm 183: 125-132, 1999.

19. Desai SD and Blanchard J: Evaluation of pluronic F127-based sustained-release ocular delivery systems for pilocarpine using the albino rabbit eye model. J Pharm Sci 87: 1190-1195, 1998.

20. Wakitani S, Okabe T, Horibe S, Mitsuoka T, Saito M, Koyama T, Nawata M, Tensho K, Kato H, Uematsu K, et al: Safety of autologous bone marrow-derived mesenchymal stem cell transplantation for cartilage repair in 41 patients with 45 joints followed for up to 11 years and 5 months. J Tissue Eng Regen Med 5: 146-150, 2011. 
21. Hodge WA, Fijan RS, Carlson KL, Burgess RG, Harris WH and Mann RW: Contact pressures in the human hip joint measured in vivo. Proc Natl Acad Sci USA 83: 2879-2883, 1986.

22. Madry H, Grün UW and Knutsen G: Cartilage repair and joint preservation: Medical and surgical treatment options. Dtsch Arztebl Int 108: 669-677, 2011.

23. Becerra J, Andrades JA, Guerado E, Zamora-Navas P, Lòpez-Puertas JM and Reddi AH: Articular cartilage: Structure and regeneration. Tissue Eng Part B Rev 16: 617-627, 2010.

24. Redman SN, Oldfield SF and Archer CW: Current strategies for articular cartilage repair. Eur Cell Mater 9: 23-32, 2005.

25. Kim HK, Moran ME and Salter RB: The potential for regeneration of articular cartilage in defects created by chondral shaving and subchondral abrasion. An experimental investigation in rabbits. J Bone Joint Surg Am 73: 1301-1315, 1991.

26. Kelly DJ and Prendergast PJ: Mechano-regulation of stem cell differentiation and tissue regeneration in osteochondral defects. J Biomech 38: 1413-1422, 2005.

27. Brittberg M, Lindahl A, Nilsson A, Ohlsson C, Isaksson O and Peterson L: Treatment of deep cartilage defects in the knee with autologous chondrocyte transplantation. N Engl J Med 331: 889-895, 1994.

28. Brittberg M: Cell carriers as the next generation of cell therapy for cartilage repair: A review of the matrix-induced autologous chondrocyte implantation procedure. Am J Sports Med 38: 1259-1271, 2010

29. Fischer J, Dickhut A, Rickert M and Richter W: Human articular chondrocytes secrete parathyroid hormone-related protein and inhibit hypertrophy of mesenchymal stem cells in coculture during chondrogenesis. Arthritis Rheum 62: 2696-2706, 2010.

30. Weiss S, Hennig T, Bock R, Steck E and Richter W: Impact of growth factors and PTHrP on early and late chondrogenic differentiation of human mesenchymal stem cells. J Cell Physiol 223: $84-93,2010$.

31. Mueller MB and Tuan RS: Functional characterization of hypertrophy in chondrogenesis of human mesenchymal stem cells. Arthritis Rheum 58: 1377-1388, 2008.

32. Williams R, Khan IM, Richardson K, Nelson L, McCarthy HE, Analbelsi T, Singhrao SK, Dowthwaite GP, Jones RE, Baird DM, et al: Identification and clonal characterisation of a progenitor cell sub-population in normal human articular cartilage. PLoS One 5: e13246, 2010.

33. Caplan AI: Why are MSCs therapeutic? New data: New insight. J Pathol 217: 318-324, 2009.

34. Johnstone B, Hering TM, Caplan AI, Goldberg VM and Yoo JU: In vitro chondrogenesis of bone marrow-derived mesenchymal progenitor cells. Exp Cell Res 238: 265-272, 1998.
35. Pelttari K, Steck E and Richter W: The use of mesenchymal stem cells for chondrogenesis. Injury 39 (Suppl 1): S58-S65, 2008.

36. Banfi A, Muraglia A, Dozin B, Mastrogiacomo M, Cancedda R and Quarto R: Proliferation kinetics and differentiation potential of ex vivo expanded human bone marrow stromal cells: Implications for their use in cell therapy. Exp Hematol 28: 707-715, 2000

37. Zhou GD, Miao CL, Wang XY, Liu TY, Cui L, Liu W and Cao YL: Experimental study of in vitro chondrogenesis by co-culture of bone marrow stromal cells and chondrocytes. Zhonghua Yi Xue Za Zhi 84: 1716-1720, 2004 (In Chinese).

38. Gardner OF, Archer CW, Alini $\mathrm{M}$ and Stoddart MJ: Chondrogenesis of mesenchymal stem cells for cartilage tissue engineering. Histol Histopathol 28: 23-42, 2013.

39. Park SS, Jin HR, Chi DH and Taylor RS: Characteristics of tissue-engineered cartilage from human auricular chondrocytes. Biomaterials 25: 2363-2369, 2004.

40. Smeriglio P, Lai JH, Yang F and Bhutani N: 3D Hydrogel Scaffolds for articular chondrocyte culture and cartilage generation. J Vis Exp: Oct 7, 2015 doi: 10.3791/53085.

41. Raghunath J, Rollo J, Sales KM, Butler PE and Seifalian AM: Biomaterials and scaffold design: Key to tissue-engineering cartilage. Biotechnol Appl Biochem 46: 73-84, 2007.

42. Kisiday J, Jin M, Kurz B, Hung H, Semino C, Zhang S and Grodzinsky AJ: Self-assembling peptide hydrogel fosters chondrocyte extracellular matrix production and cell division: Implications for cartilage tissue repair. Proc Natl Acad Sci USA 99: 9996-10001, 2002

43. Fisher JP, Jo S, Mikos AG and Reddi AH: Thermoreversible hydrogel scaffolds for articular cartilage engineering. J Biomed Mater Res A 71: 268-274, 2004.

44. Yasuda A, Kojima K, Tinsley KW, Yoshioka H, Mori Y and Vacanti CA: In vitro culture of chondrocytes in a novel thermoreversible gelation polymer scaffold containing growth factors. Tissue Eng 12: 1237-1245, 2006.

45. Kaneko Y, Nakamura S, Sakai K, Kikuchi A, Aoyagi T, Sakurai Y and Okano T: Synthesis and swelling-deswelling kinetics of poly(N-isopropylacrylamide) hydrogels grafted with LCST modulated polymers. J Biomater Sci Polym Ed 10: 1079-1091, 1999.

c) () $\odot$ This work is licensed under a Creative Commons c. AY NO ND Attribution-NonCommercial-NoDerivatives 4.0 International (CC BY-NC-ND 4.0) License. 